\title{
La lipolyse dans le lait refroidi
}

\author{
par \\ Simonne KUZDZAL-SAVOIE, J. E. AUCLAIR, R. MOURGUES \\ et D. LANGLOIS
}

Station Centrale de Recherches Laitières et de Technologie des Produits Animaux, I.N.R.A.C.N.R.Z. - 78350 Jouy-en-Josas (France)

L'utilisation de plus en plus répandue du refroidissement du lait à la ferme permet de prolonger jusqu'à $48 \mathrm{~h}$ le délai qui sépare la sécrétion du lait de sa livraison à la laiterie. A la laiterie même, il arrive souvent que le lait soit encore stocké au froid $\left(4-6^{\circ} \mathrm{C}\right)$ pendant 1 ou 2 jours avant de subir le traitement thermique de pasteurisation ou de stérilisation.

Peu visible autrefois lorsque le lait était traité pratiquement dans les $24 \mathrm{~h}$ qui suivent sa récolte, le phénomène de lipolyse est devenu un problème majeur de l'industrie laitière européenne actuelle, après avoir préoccupé les chercheurs et les techniciens américains il y a 20 ou 25 ans.

La raison première est l'apparition fréquente dans un lait maintenu à température relativement basse d'un défaut d'arôme, la " rancidité ". Ce défaut, sous des formes diverses (goût de rance, de piquant, goût de savon), peut également être observé dans les produits laitiers (crème, beurre, fromages, poudres diverses).

Quelle est la nature de ce phénomène ? Quelles en sont les causes ? Quels sont les facteurs qui le favorisent ? Comment le mesurer ? Comment l'éviter ?

Ce sont là les principales questions auxquelles les informations scientifiques, technologiques et techniques rassemblées à l'occasion du Symposium sur la lipolyse tenu à Cork du 4 au 7 mars 1975, apportent un certain nombre de réponses.

\section{I. - Le PHenOMENE DE LA LiPOLYSE. ROLE DE LA LIPASE ET DES FACTEURS PHYSIOLOGIQUES}

Dans les conditions optimales $\left(37^{\circ} \mathrm{C}, \mathrm{pH} 8,5\right)$, la lipase, toujours présente dans le lait, peut hydrolyser suffisamment de triglycérides 
en quelques minutes pour qu'apparaisse un fort défaut organoleptique de rancidité. Même à température relativement basse et au $\mathrm{pH}$ normal du lait, l'hydrolyse est encore rapide. Pourquoi donc le lait ne devient-il pas toujours lipolysé ? Sans doute parce qu'un ou plusieurs facteurs empêchent ou au moins gênent l'action de la lipase.

\section{A. - La lipase. Les enzymes lipolytiques}

Lors de sa sécrétion, le lait de vache contient toujours une faible quantité d'acides gras libres (de l'ordre de 0,25 milliéquivalents pour $100 \mathrm{~g}$ de matière grasse). Ceux-ci résultent vraisemblablement d'une synthèse incomplète des glycérides et ne proviennent pas de la lipolyse.

Par ailleurs, on sait depuis longtemps que le lait contient une lipase. Considérée généralement comme inactive dans le lait fraîchement trait, cette lipase, pensait-on, devenait " active " sous l'effet conjugué du refroidissement, de l'agitation du lait, des rentrées d'air dans les machines à traire, de la formation de mousse, etc., d'où le terme de lipolyse "induite » introduit pour qualifier ce phénomène, observé le plus souvent sur des laits de mélange, et à l'origine du défaut organoleptique de rancidité.

Cependant certains laits deviennent "rances " rapidement sous l'effet d'un simple séjour à basse température. Les études poursuivies sur les laits individuels ont mis nettement en évidence ce phénomène de « lipolyse spontanée ». La lipolyse spontanée est difficile à distinguer de la lipolyse induite dans les laits de grand mélange (Kuzdzal-Savoie et Mocquot, 1960). En 1957, Tarassuk et Frankel ont proposé l'hypothèse suivante : le lait de vache contient deux lipases (alcalines), présentes dans la phase aqueuse du lait fraîchement trait. L'une d'elles, la lipase de membrane, présente à relativement forte concentration dans les laits « naturellement actifs " (laits de fin de lactation par exemple), est irréversiblement adsorbée sur la membrane des globules gras lors du refroidissement du lait. L'autre lipase, la lipase «plasmatique " associée avec la caséine, ne peut devenir active qu'à la suite d'un traitement « d'activation ».

Les termes de "lipolyse induite " et de "lipolyse spontanée " s'accordaient bien avec la théorie de Tarassuk. Malheureusement la lipase de membrane n'a pas été isolée (bien que récemment il ait été montré qu'une proportion plus élevée d'activité lipasique liée à la membrane était observée dans les laits montrant une lipolyse spontanée par rapport aux laits normaux), et par la suite on préféra parler des « enzymes lipolytiques » sans les définir. Cependant les travaux se développèrent et la notion d'une multiplicité de lipases dans le lait s'imposa. On distingua dans le lait, une lipase "vraie ", hydrolysant les triglycérides en émulsion (et hydrolysant d'ailleurs plus rapidement les triglycérides les plus courts) et une « lipoprotéine- 
lipase »(Korn, 1962) hydrolysant également les triglycérides en émulsion mais à condition cependant que ceux-ci soient complexés avec les lipoprotéines du sérum. En outre, la présence d'une lipase acide était suggérée (Albrecht et Jaynes, 1955).

Dans le lait, peuvent enfin exister des lipases d'origine bactérienne ou fongique, dont on connaît la grande thermostabilité.

Des éclaircissements sur la nature des enzymes lipolytiques existant dans le lait de vache ont pu être obtenus grâce aux travaux récents de Downey* en Irlande et d'Olivecrona* en Suède, dont les résultats ont été exposés au Symposium de Cork.

D'une part il a été précisé que les enzymes sécrétées par les micro-organismes ne peuvent jouer un rôle dans le lait, c'est-à-dire contribuer par exemple à l'accroissement de la lipolyse, que si le nombre de ces micro-organismes atteint au moins $10^{7}$ par ml. D'autre part des doutes ont été émis sur l'existence d'une lipase acide active dans le lait.

Ainsi l'activité lipolytique dans le lait serait essentiellement fournie par les enzymes lipolytiques alcalines et, ce qui est fondamental, les travaux les plus récents de Olivecrona* et Downey* montrent que la distinction faite antérieurement entre les activités revenant à la lipase et celles revenant à la lipoprotéine-lipase devrait être remise en cause.

L'inhibition par le chlorure de sodium ou par le sulfate de protamine qui est utilisée pour distinguer ces deux activités enzymatiques ne donne de résultats valables que si la préparation d'enzyme est pré-incubée avec l'inhibiteur. Or de telles conditions n'ont pas toujours été réalisées dans les expériences de certains auteurs. Par contre, l'étude du pH optimal, de la stabilité à la chaleur, aux rayons U.V. ou aux rayons $\gamma$, de la sédimentation, de la filtration sur gel, des propriétés chromatographiques, des conditions d'élimination des enzymes des micelles de caséine sous l'effet de l'héparine, tout cela converge vers la conviction qu'il n'existe qu'une enzyme active vis-àvis des triglycérides en émulsion, précisément la lipoprotéine-lipase (E.C.3.1.1.3.). On remarquera que le tissu mammaire ne contient également qu'une enzyme lipolytique : une lipoprotéine-lipase précisément. Cette enzyme n'est active qu'en un site défini : à la surface de l'endothélium capillaire. Le mode de passage de l'enzyme à travers le tissu séparant la surface de l'endothélium capillaire de la lumière alvéolaire reste cependant inconnu.

Olivecrona* suggère la synthèse dans la cellule sécrétrice de la glande mammaire d'un pro-enzyme inactif qui peut aussi être sécrété dans le lait.

Le signe* placé après certains noms d'auteurs indique que les travaux cités, exposés au Symposium de Cork, ne sont pas encore publiés. 


\section{B. - Les co-facteurs}

Pour atteindre une activité optimale la lipase du lait requiert un co-facteur.

Schipper* et Jellema* ont montré que les lipoprotéines du plasma sanguin constituent une source de co-facteur. L'addition de 1 p. 100 de sérum bovin à du lait entraîne une lipolyse immédiate. Par ailleurs les lipoprotéines de plasma sanguin ont été reconnues dans du lait manifestant une lipolyse spontanée.

Ainsi l'existence dans le lait d'un activateur lipoprotéinique (peptide activateur ?) pour la lipase explique probablement l'apparition de la lipolyse spontanée.

L'alimentation, le stade de lactation jouent un rôle dans l'apparition de la rancidité. Dès qu'une baisse de production survient, un risque de lipolyse est latent. Existe-t-il un rapport entre ces observations et le taux de lipoprotéines du sérum dans le lait ? Des travaux restent à faire dans ce domaine.

La corrélation observée entre le nombre des cellules et le développement de la rancidité pourrait être due à la présence, dans le lait à forte teneur en cellules, de composés du plasma sanguin.

Le passage des constituants du sang dans le lait apparaît ainsi comme le phénomène principalement en cause dans le développement de la lipolyse spontanée et l'apparition de la rancidité. Les travaux de Jellema* et Schipper* et de Driessen et Stadhouders appuient ces conclusions.

Cependant ni la nature du "peptide activateur » ni les conditions de son action n'ont été définies. Downey* et Murphy* ont étudié la nature de la liaison entre les micelles de caséine et la lipase. Le glycomacropeptide joue probablement un rôle dans la stabilité de cette liaison.

Death* et Fitzgerald* ont d'autre part mis en évidence la présence dans le lait écrémé d'inhibiteurs de la lipolyse. Thermostables et dialysables, ces facteurs sont présents en quantités variables dans les laits et déterminent peut-être dans une certaine mesure une susceptibilité variable des laits à la lipolyse.

\section{C. - La membrane}

La membrane des globules gras protège les glycérides de l'action de la lipase : le contact entre l'enzyme et le substrat ne peut se faire.

Grâce aux observations faites à l'aide du microscope électronique sur des cellules sécrétrices de la glande mammaire de chèvre et de vache, Wooding* apporte des informations nouvelles sur l'origine de la membrane des globules gras. Cet auteur montre le rôle important joué par les vésicules de Golgi. Au cours de leur élaboration dans la cellule sécrétrice, les gouttelettes lipidiques, puis les globules gras 
se trouvent progressivement entourés par de nombreuses vésicules de Golgi qui se placent en position adjacente. Lors de l'excrétion dans la lumière alvéolaire le globule gras est alors entouré d'une enveloppe dérivée de la membrane golgienne enserrant parfois, entre la matière grasse et la membrane, une zone aqueuse. Cette zone aqueuse peut occasionnellement être très large et contenir alors des organites cytoplasmiques identifiables (reticulum endoplasmique, mitochondries).

Lorsque le globule se trouve dans la lumière alvéolaire, il est entouré d'une couche périphérique qui lui est propre (constituée de phospholipides et de protéines), surmontée de fragments de membrane externe dérivée de la membrane des vésicules de Golgi.

La teneur en protéines et en phospholipides de la membrane des globules gras a été analysée récemment par Anderson et al. (1972) ; la composition de la membrane varie en fonction de la race, de l'alimentation et du stade de lactation de l'animal. Le froid modifie également la composition de la membrane ; on peut observer après conservation du lait au froid une perte de près de $1 / 3$ du matériel membranaire. Parfois une adsorption de constituants provenant de la phase aqueuse se produit : ainsi des micelles de caséine adsorbées sur la membrane peuvent entraîner une fraction de lipase. Mais la nature exacte des échanges reste inconnue. Cependant des modifications dans le matériel enzymatique de la membrane ont été mises en évidence.

Les traitements mécaniques que subit le lait dénaturent la membrane des globules gras et créent des risques de "lipolyse induite ».

Cependant un problème nouveau est apparu. Dans le colostrum de vache Driessen et Stadhouders ont mis en évidence une lipase dont l'activité n'est pas stimulée par les lipoprotéines et qui n'est pas liée aux micelles de caséine.

Il pourrait s'agir d'une lipase autre que la lipoprotéine-lipase, semblable par exemple à la lipase activée par les sels biliaires récemment isolée du lait humain par Olivecrona et al. (1973) à côté de la lipoprotéine-lipase.

Bien des questions devront trouver une réponse avant que le mécanisme de la lipolyse par la lipase naturelle du lait soit parfaitement élucidé.

\section{II. - LES DEFAUTS ORGANOLEPTIQUES LA LIPOLYSE DANS LE LAIT ET LES PRODUITS LAITIERS}

Il a été démontré que les acides gras comportant une très courte chaîne (acides formique, acétique, propionique) ou une très longue chaîne (acides comportant 14 à 18 atomes de carbone) ne sont pas en 
cause dans l'apparition de la rancidité. Par contre les acides gras comptant 4 à 12 atomes de carbone, jouent un rôle important et contribuent d'une manière pratiquement égale à l'apparition du défaut. L'acide butyrique n'est pas seul en cause comme on le croit encore trop souvent.

Des informations manquent encore pour que puisse être reconstitué un "étalon de référence " caractérisant le défaut de rance.

Cependant les travaux récents (1974) de Paulet et al., ont permis de connaître l'origine du « goût de savon » qui constitue une des modalités de la rancidité. Le goût de savon est dû à la présence d'acide laurique ou d'acide caprique libre. Les seuils de perception du goût de savon dans l'eau sont de $25 \mathrm{ppm}$ et $50 \mathrm{ppm}$ respectivement pour les deux acides. La perception du goût de savon exige une quantité d'acide gras libre supérieure à la quantité correspondant au seuil de perception de l'acide.

Dans l'huile, plus la chaîne de l'acide gras est longue, plus est élevé le seuil de perception : 2,5 ppm pour l'acide caproïque, 700 ppm pour l'acide laurique.

Une acidité titrable de la phase grasse inférieure à 1,5 $\mathrm{m}$ équivalent d'acides gras pour $100 \mathrm{~g}$ de matière grasse n'entraîne généralement pas de rancidité détectable ; mais si l'acidité titrable dépasse $1,5 \mathrm{~m}$ équivalent la rancidité est généralement perçue. Cette acidité titrable correspond à une concentration en acide caprique sensiblement égale au seuil de perception de cet acide.

Il semble que la quantité d'acides gras entraînant la perception du défaut de rancidité soit plus faible que celle entraînant la perception du goût de savon. Cependant le défaut de rance est encore mal défini. Est-il dû uniquement à la présence de certains acides gras ou est-il dû à la présence simultanée de certains acides gras et d'autres composés ? Le pH du milieu exerce une influence décisive sur la perception du défaut de rance. Tuckey et Stadhouders en avaient déduit en 1968 une méthode permettant d'accroître la sensibilité de l'examen organoleptique du lait par simple addition d'une solution d'acide citrique. Un tri préliminaire des laits destinés à la fermentation basé sur cette méthode permettait ainsi d'éviter l'apparition de la rancidité dans les laits acidifiés.

Il est cependant bien établi que l'accroissement des acides gras libres entraîne l'apparition des défauts organoleptiques. La teneur en acides gras libres constitue donc un critère valable pouvant être utilisé comme base d'un contrôle de qualité.

\section{Lipolyse dans le lait}

Les analyses systématiques effectuées en Hollande et en Irlande ont révélé l'existence d'une variation saisonnière de l'intensité de la lipolyse des laits livrés aux laiteries. Peu importante pendant la vie 
au pâturage des vaches laitières, la lipolyse atteint un niveau plus élevé pendant la période hivernale.

Par ailleurs, l'influence du stade de lactation est très nette. En fin de lactation, la teneur en acides gras libres de la plupart (mais non de la totalité) des laits individuels s'accroît et il est probable que le stade de lactation intervient pour expliquer au moins partiellement la variation saisonnière de la teneur en acides gras libres du lait récolté. Toute réduction occasionnelle de production, au niveau de l'animal, s'accompagne d'un accroissement de la lipolyse.

Il est possible que l'alimentation à l'herbe joue un rôle inhibiteur, non expliqué, vis-à-vis du développement de la lipolyse.

Les conclusions antérieures sur l'apparition du défaut de rance dans le lait (Kuzdzal-Savoie et Mocquot, 1960) sont donc renforcées par les observations nouvelles relatives à la teneur en acides gras libres de la matière grasse du lait : influence de la saison, du stade de lactation, de l'individualité des vaches laitières, etc.

\section{Lipolyse dans le beurre}

Au cours de l'année le beurre fabriqué a une acidité libre de la phase grasse qui reflète celle du lait à partir duquel il est fabriqué.

A basse température $\left(-10^{\circ} \mathrm{C}\right.$ ou $\left.-20^{\circ} \mathrm{C}\right)$ l'acidité libre de la phase grasse reste constante, mais les beurres qui présentent la plus faible acidité libre sont aussi les beurres qui manifestent la meilleure aptitude à la conservation.

A $15^{\circ} \mathrm{C}$ par contre, l'acidité libre de la phase grasse évolue, plus particulièrement pour les beurres non salés. En Irlande, on a constaté que, si les beurres de crème douce non salés sont généralement les plus vulnérables, ceux fabriqués en mai et juin présentent une bonne résistance à l'altération. Les beurres de crème acide non salés fabriqués en décembre et janvier sont plus vulnérables que ceux fabriqués à une autre période de l'année.

Toute une série de déterminations de l'acidité libre de la phase grasse effectuées par une entreprise belge sur des beurres d'origine variée et provenant des différents pays d'Europe ou du Monde a permis de montrer qu'il y avait également une influence géographique. Les beurres présentant la plus faible lipolyse proviennent généralement de régions où le climat particulièrement doux et humide permet aux vaches laitières de bénéficier d'une vie prolongée au pâturage (Basse-Normandie par exemple).

A la lipolyse due à l'action des enzymes lipolytiques naturelles du lait s'ajoute le risque de lipolyse dû à la présence des micro-organismes psychrotrophes.

Alors que les enzymes lipolytiques naturelles du lait sont détruites par un traitement thermique modéré $\left(74^{\circ} \mathrm{C}\right.$ pendant $15 \mathrm{~s}$ par 
exemple) les enzymes lipolytiques sécrétées par les micro-organismes psychrotrophes présentent une thermorésistance prononcée. Même lorsque les micro-organismes qui produisent ces enzymes sont détruits, les enzymes elles-mêmes continuent d'exercer leur action.

Il convient donc de limiter au maximum le développement des micro-organismes psychrotrophes. En Hollande, un traitement de thermisation $\left(65^{\circ} \mathrm{C}, 5 \mathrm{~s}\right.$ ou $\left.63^{\circ} \mathrm{C}, 10 \mathrm{~s}\right)$ est appliqué à tout lait arrivant à la laiterie et non transformé dans les $24 \mathrm{~h}$ qui suivent. Ce traitement permet de réduire le développement des psychrotrophes.

Kishonti et Sjostrom (1970) ont étudié la thermorésistance de certaines enzymes lipolytiques produites par les micro-organismes. Travaillant sur 60 souches de bactéries psychrotrophes du genre Pseudomonas, Alcaligenes, Aerobacter et autres isolées de laits réfrigérés, à la ferme, ces auteurs ont montré que 30 p. 100 des souches produisent des enzymes qui supportent un traitement thermique de $2 \mathrm{mn}$ à $90^{\circ} \mathrm{C}$ sans perdre plus de $75 \mathrm{p} .100$ de leur activité initiale. Kishonti* a indiqué au Symposium de Cork, qu'en Suède, dans les beurreries, la crème subit un traitement thermique prononcé $\left(108^{\circ} \mathrm{C}\right.$ $\left.110^{\circ} \mathrm{C}\right)$.

Les lipases de certaines souches exigent un traitement de 5 à $10 \mathrm{mn}$ à $130^{\circ} \mathrm{C}$ pour ramener l'activité à $1 \mathrm{p} .100$ de la valeur initiale. Un traitement U.H.T. à $150^{\circ} \mathrm{C}$ est nécessaire pour détruire ces enzymes.

La lipolyse due aux micro-organismes a peu d'effet dans le lait de consommation ou même le beurre, sauf si la prolifération des micro-organismes psychrotrophes dépasse $10^{7}$ par $\mathrm{ml}$.

Son terrain de prédilection est le fromage.

\section{Lipolyse dans le fromage}

La lipolyse dans le fromage est un phénomène normal. A chaque variété de fromage correspond un taux de lipolyse optimal, au-delà duquel un défaut de goût apparaît : goût de rance, ou goût de savon.

La lipolyse dans le fromage n'est pas préférentielle vis-à-vis de tel ou tel acide, et la répartition des acides gras libres est assez similaire à la répartition des acides gras estérifiés. Ainsi, si l'on détermine la quantité d'acide caproïque libre, on peut connaître approximativement la quantité d'acides gras libres totaux, et donc l'importance relative du phénomène de lipolyse par rapport aux autres phénomènes biochimiques de l'affinage.

La quantité d'acide caproïque est aisée à déterminer lors de l'analyse des acides libres volatils par chromatographie en phase gazeuse. La quantité d'acide caproïque libre au-delà de laquelle le défaut organoleptique apparaît peut-être déterminée pour chaque variété de fromage (par exemple $8 \mathrm{mg}$ pour $100 \mathrm{~g}$ de matière sèche 
pour l'Emmental, $14 \mathrm{mg}$ pour le Gruyère de Comté, $25 \mathrm{mg}$ pour le Camembert, etc.).

\section{III. - FACTEURS TECHNOLOGIQUES QUI FAVORISENT LA LIPOLYSE}

On connaît depuis longtemps l'influence du matériel de traite sur le degré de lipolyse du lait. Tolle et Heeschen ont observé que la teneur en acides gras libres du lait double lorsque l'on passe de la traite mécanique en pots-trayeurs à un système comportant un pipeline amenant directement le lait au tank de ferme. Tous les auteurs sont également d'accord sur l'avantage que présentent les installations de traite " à ligne basse » par rapport aux installations à " ligne haute ».

Toutefois certains auteurs estiment que le mal est déjà fait avant l'entrée du lait dans le pipeline, et que la lipolyse se produirait dès le faisceau-trayeur et le tuyau à lait. On a constaté également que l'utilisation de canalisations de plus grand diamètre permet de réduire les risques de lipolyse. L'accent est mis aussi sur le rôle néfaste du moussage du lait dans les installations de traite, moussage dû aux entrées d'air, aux remontées de lait dans les tuyauteries, ainsi qu'aux pompes tournant presque à vide. La façon dont le lait pénètre dans le releaser et l'introduction du lait dans le tank lui-même sont également des facteurs influant sur la lipolyse. Tous ces points pourraient faire l'objet de recommandations dans une norme internationale.

Il semble donc que la lipolyse soit un problème qui puisse, en grande partie, être résolu par le producteur lui-même. Du bon sens et une bonne information du producteur amélioreraient grandement la situation. Quant au tank de ferme lui-même, on reconnaît qu'il ne provoque pas, ou pratiquement pas de lipolyse.

\section{IV. - DETERMINATION DE LA LIPOLYSE DANS LE LAIT}

Deux objectifs sont possibles. On peut vouloir déterminer le degré d'altération à un moment donné et mesurer alors la quantité d'acides gras libres présents. On peut également vouloir connaître les risques ultérieurs de lipolyse et déterminer l'activité lipolytique potentielle du lait à un moment donné. 


\section{1) Détermination des acides gras libres}

Il existe de nombreuses méthodes de détermination des acides gras libres. Ces méthodes permettent toutes d'évaluer une variation d'intensité de la lipolyse, mais elles ne conduisent pas toutes aux mêmes résultats.

Le dosage acidimétrique des acides gras libres contenus dans la matière grasse ou entraînés avec elle est une technique très répandue. La matière grasse est isolée soit par écrémage du lait, fabrication du beurre, fusion et centrifugation de celui-ci, soit par l'action combinée de l'agitation, de la chaleur et d'un détergent (méthode B.D.I.) (Thomas et al., 1955 ; Jamotte, 1967, 1972).

Dans ces deux procédés d'isolement de la matière grasse, le dosage des acides gras libres est effectué selon la méthode décrite dans la norme internationale FIL-IDF $\mathrm{n}^{\circ}$ 6, 1959.

Les acides gras libres peuvent être également extraits et dosés directement à partir du lait ou de la crème.

Par la méthode de Frankel et Tarassuk (1955), on dose les acides gras libres sur un simple extrait éthéro-alcoolique du lait additionné de chlorure de sodium. Les méthodes de Harper et al. (1956) et de Bachmann (1960) sont très voisines. Elles utilisent toutes deux la chromatographie sur colonne d'acide silicique, avec acidification à pH 2,0 du lait ou de la crème. La différence essentielle entre ces deux méthodes est la composition du solvant d'élution : chloroforme contenant 5 p. 100 de butanol (Harper), chloroforme pur (Bachmann). Cette différence de polarité du solvant d'élution, permet de ne recueillir par la méthode Bachmann que les acides gras libres comptant au moins 6 atomes de carbone.

Les valeurs obtenues par l'emploi des méthodes de Frankel et Tarassuk et de Harper et al. sont beaucoup plus élevées que les valeurs obtenues par les autres méthodes. Ceci a été confirmé par Jamotte (1967) et les résultats obtenus par Stadhouders et al. (1967) conduisent aux mêmes conclusions.

La comparaison des résultats est d'ailleurs compliquée par la différence dans l'expression choisie : en milliéquivalents d'acides gras par litre de lait ou en milliéquivalents d'acide gras pour $100 \mathrm{~g}$ de matière grasse.

En effet, non seulement les acides gras libres à chaîne courte sont préférentiellement entraînés dans le babeurre mais encore 30 p. 100 environ des acides libres à longue chaîne sont perdus lors de la fabrication du beurre et se rassemblent dans la phase aqueuse.

Une méthode colorimétrique antérieurement appliquée au dosage des acides gras libres du sérum sanguin (Mc Kenzie et al., 1967) a été récemment adaptée au dosage des acides gras libres du lait, selon deux modalités différentes : utilisation de rhodamine B (Nakai et al., 
1970) ou de rhodamine G (Chakrabarty et al., 1969 ; Kason et al., 1972).

La complexité de la méthode colorimétrique citée, dont on a effectué une étude approfondie rend difficile son application à Ia détermination des acides gras libres du lait malgré les simplifications proposées. De plus les résultats obtenus par cette méthode apparaissent faibles comparés aux résultats obtenus par la méthode BDI par exemple.

Une méthode analytique de dosage des acides gras libres du lait a été décrite par Lindquist et al. en janvier 1975. Cette méthode comporte une extraction préliminaire des lipides totaux par un mélange d'isopropanol, d'heptane et d'acide sulfurique dilué, suivie d'un dosage colorimétrique en présence de rouge de phénol. L'appareillage utilisé est un Autoanalyzer II.

La méthode automatique préconisée par Lindquist conduit a des résultats environ 20 p. 100 plus élevés que les résultats obtenus par la méthode BDI à partir des mêmes échantillons de lait.

Schipper (document IDF-FIL $n^{\circ}$ 82) a montré que la différence entre l'acidité libre du lait entier et l'acidité libre décelée dans le lait écrémé correspondait approximativement à l'acidité libre de la phase grasse. L'origine de l'acidité libre du lait écrémé reste cependant mal définie.

Une étude approfondie de la signification précise de chacune des méthodes, de ce qu'elles dosent ou ne dosent pas, permettrait de justifier le choix de l'une d'entre elles en vue d'une normalisation et d'établir sur des bases solides une comparaison des méthodes.

\section{2) Mesure de l'activité des enzymes lipolytiques}

L'activité des enzymes lipolytiques du lait peut être mesurée par la quantité d'acides gras libérés en fonction du temps à partir d'un substrat défini.

Jusqu'à maintenant on distinguait dans le lait la mesure de l'activité due à la lipase proprement dite de l'activité due à la lipoprotéine-lipase.

Tous les éléments d'information relatifs à la détermination distincte de ces deux activités lipolytiques se trouvent réunis dans le document 82 : "Lipolyse dans le lait refroidi en vrac ", chapitre V (Downey et Moore).

L'activité lipasique est mesurée à pH 8,5 dans une solution de tributyrine émulsionnée à l'aide de gomme arabique en présence de chlorure de sodium et de chlorure de magnésium. L'activité lipasique est mesurée par titration continue avec une solution basique dans des conditions qui maintiennent le $\mathrm{pH}$ constant. L'addition d'hydroxyde de sodium peut être manuelle ou automatique. La droite repré- 
sentant le volume d'hydroxyde de sodium ajouté en fonction du temps a une pente d'autant plus forte que l'activité lipolytique est plus prononcée. Celle-ci s'exprime en $\mu$ moles ou microéquivalents d'acides gras libérés par minute et par $\mathrm{ml}$ de lait (de 0,5 à $2 \mu \mathrm{eq} / \mathrm{ml} / \mathrm{mn}$ ). L'addition de chlorure de sodium dans le mélange incubé permet de libérer la lipase des micelles de caséine et assure ainsi la détection de l'activité lipasique totale du lait. Par ailleurs le chlorure de sodium utilisé inhibe l'activité de la lipoprotéine-lipase.

L'activité de la lipoprotéine-lipase du lait est déterminée par la mesure des acides gras libres formés par l'action du lait sur les substrats constitués par des émulsions de triglycérides activés par pré-incubation avec du sérum sanguin ou des lipoprotéines du sérum. L'activité de la lipoprotéine-lipase dans le lait varie de 0,4-1,0 à 6-10 microéquivalents d'acides gras $/ \mathrm{ml} / \mathrm{mn}$.

Tout ce qui vient d'être exposé risque d'être remis en cause puisque selon les récentes études exposées à Cork lors du Symposium sur la lipolyse, la lipase et la lipoprotéine-lipase sont en fait la même enzyme ; la mesure de la seule activité de la lipoprotéine-lipase constitue donc la détermination essentielle.

Toutefois il serait nécessaire de mesurer également l'activité lipolytique due à l'enzyme activée par les sels biliaires et dont la présence dans le colostrum de vache a été établie par Driessen et Stadhouders.

Enfin, il semble justifié de déterminer, en outre, l'activité estérasique du lait. La méthode britannique de détermination de l'activité lipolytique basée sur la modification d'un substrat (acétate d'indoxyle), avec formation d'un composé coloré (indigo) pourrait sans doute être utilisé avec profit pour la détermination de l'activité estérasique dans le lait.

Un autre problème important consiste à différencier l'activité lipolytique due aux enzymes naturelles du lait de l'activité lipolytique due aux enzymes bactériennes ou fongiques. Lawrence et al. (1967) ont posé les bases d'une telle différenciation. Une exploitation des possibilités analytiques existantes mériterait d'être proposée.

En conclusion, la mise au point d'une méthode pratique de détermination de l'activité lipolytique du lait nécessite quelques travaux supplémentaires.

\section{CONCLUSION}

Le Symposium sur la lipolyse tenu à Cork du 5 au 7 mars 1975 a permis de faire le point de nos connaissances actuelles sur ce problème dont les aspects pratiques ne peuvent être séparés des aspects fondamentaux. 
Des efforts doivent être poursuivis sur le plan de la recherche en vue de préciser les conditions d'activité de la lipoprotéine-lipase du lait, la nature exacte de la lipase de colostrum non liée à la caséine, l'existence d'une corrélation entre la susceptibilité à la lipolyse des laits individuels et le contenu en lipoprotéines, le mécanisme d'initiation de la lipolyse spontanée par le froid, la répartition entre crème, sérum, caséine, des enzymes lipolytiques dans les laits actifs.

La méthodologie exige de nouvelles investigations en vue de préciser la signification exacte des différentes méthodes afin qu'un choix argumenté soit possible.

Le plus important, dans l'immédiat, reste cependant de promouvoir l'effort d'éducation qui devrait permettre une réduction sensible des problèmes auxquels se heurte actuellement l'industrie laitière européenne.

\section{S u m m a r y}

\section{LIPOLYSIS IN REFRIGERATED MILK}

Following the Symposium organised by I D F at Cork, Ireland, in March 1974 on this subject, the authors present a review of the problem of lipolysis.

They review the role of lipolytic enzymes, of co-factors such as blood plasma lipoproteins, and of the fat globule membrane on the intensity of lipolysis. The organoleptic defects caused by lipolysis in milk, butter and cheese are examined and the influence of the milking machine is recalled as an important factor in the development of lipolysis.

Finally the methods for the detection of free fatty acids and for the determination of the activity of the lipolytic enzymes are reviewed.

\section{Références}

Albrecht (T. W.), Jaynes (H. O.) (1965). - Milk lipase. J. Dairy Sci., 38, 137-146.

Anderson (M.), Cheeseman (G. C.), Knight (D. J.), Shipe (W. F.) (1972). - The effect of ageing cooled milk on the composition of the fat globule membrane. J. Dairy Res., 39, 95-105.

BachmanN (B.) (1960). - Beitrag zur kemtnis der lipolytischen Fettspaltung (Ranzigkeit) in milch und Käse. Prom. N.I. 3043, Zurich.

Chakrabarty (M. M.), Bhattacharyya, Kundu (M. K.) (1969). - A simple photometric method for microdetermination of fatty acids in lipids. J. Amer. Oil Chem. Soc., 46, 473-475.

Driessen (F. M.), Stadhouders (J.) (1974). - A study of spontaneous rancidity. Neth. Milk Dairy J., 28, 130-145. 
Frankel (E. N.), TARASSuK (N. P.) (1955). - An extraction-titration method for the determination of free fatty acids in rancid milk and cream. J. Dairy Sci., 38, 751-763.

Harper (W. J.), Schwartz (D. P.), El Hagaraway (I. S.) (1956). - A rapid silicagel method for measuring total free fatty acids in milk. J. Dairy Sci., 39, 46-50.

Jamotte (P.) (1967). - Degradation de la matière grasse par lipolyse. Le Lait, $\mathrm{n}^{\circ} 461-462,25-42$.

JAMotTe (P.) (1972). - Note concernant la méthode B.D.I. utilisée à Gembloux. (Document inédit).

Kason (C. M.), Pavamani (I. V. P.), NaKai (S.) (1972). - Simple test for milk lipolysis and changes in rancidity in refrigerated pasteurized milk. J. Dairy Sci., 55, 1420-1423.

Kishonti (E.), SJostrom (G.) (1970). - Influence des lipases et protéases thermorésistantes des bactéries psychrotrophes sur la qualité des produits laitiers. Congr. Intern. Lait., Sydney, 1 F, p. 516.

KoRn (E. D.) (1962), - The lipoprotein-lipase of cow's milk. J. Lipid Res., 3, 137-146.

Kuzdzal-Savoie (S.), Mocouot (G.) (1960). - Observations sur les qualités organoleptiques du lait. Ann. Technol. (I.N.R.A.), 9, 5-52.

Lawrence (R. C.), Fryer (T. F.), Reiter (B.) (1967). - Rapid method for the quantitative estimation of microbial lipases. Nature, 213, $\mathrm{n}^{\circ} 5082,1264-1265$.

Lindeuist (B.), Roos (T.), Fujita (H.) (1975). - Auto-analyzer determination of free fatty acids in farm milk. Modification of present methods to simplify transportation of the sample. Milchwissenschaft, 30, 12-17.

Mc Kenzie (R. D.), Blohm (T. R.), Auxier (E. M.), Luther (A. C.) (1967). - Rapid colorimetric method or free fatty acids. J. Lipid Res., 8, 589-597.

Nakai (S.), Perrin (J. J.), Wright (V.) (1970). - Simple test for lipolytic rancidity in milk. J. Dairy Sci, 53, 537-540.

Olivecrona (T.), Hernell (O.), Egelrud (T.), Billstrom (A.), Helander (H.), SAMUELSON (G.), FredrikzoN (B.) (1973). - Studies on the gastric lipolysis of milk lipids in suckling rats and in human infants. In Dietary lipids and Postnatal development, Galli, Jacini, Pecile, ed., Milan, p. 77-89.

Paulet (G.), Mestres (G.), Cronenberger (L.) (1974). - Le goût de savon dans les produits alimentaires : effet de la lipase du poivre blanc. Rev. Franç. Corps Gras, 21, 611-616.

Stadoudhers (J.), Tuckey (S. L.), RaAdsveld (C. W.) (1967). - Comparaison of certain methods for the determination of fat hydrolysis in milch. Neth Milk Dairy J., 21, 150-157.

Tarassuk (N. P.), Frankel (E. N.) (1957). - The specificity of milk lipase. IV. Partition of the lipase system in milk. J. Dairy Sci., 40, 418-430.

Thomas (E. L.), Nielsen (A. J.), Olson (J.C.) (1955). - Hydrolytic rancidity in milk : a simplified method for estimating the extent of its development. Amer. Milk Review, 17, 50, 85.

TUCKEy (S. L.), StadHouders (J.) (1967). - Increase in the sensitivity of the organoleptic detection of lipolysis in cow's milk by culturing or direct acidification. Neth. Milk Dairy J., 21, 158-165. 